\title{
Legionella contamination of waterlines?
}

\author{
Prevalence of legionella waterline contamination and Legionella pneumophila antibodies in general dental \\ practitioners in London and rural Northern Ireland C. L. Pankhurst, W. Coulter, J. J. Philpott-Howard, \\ T. Harrison, F. Warburton, S. Platt, S. Surman and S. Challacombe Br Dent J 2003; 195: 591-594
}

\section{Objectives}

To determine the prevalence of legionellae in dental unit waterlines (DUWL) in general dental practices in London and rural Northern Ireland and whether the organism occurs at a high enough frequency and magnitude in DUWL to represent a threat to dentists' health.

\section{Materials and method}

Two hundred and sixty six (166 London, 100 Northern Ireland) randomly selected dental surgeries were recruited. Standardised $250 \mathrm{ml}$ water samples were taken from the DUWL and 1 litre samples from the surgery cold water tap to measure the prevalence of legionellae. The dentists provided a blood sample for legionella serology.

\section{Results}

The prevalence of legionellae was very low (0.37\%). Legionellae were not isolated from DUWL or surgery basin taps in Northern Ireland. Legionella spp were isolated from the DUWL and surgery basin of one practice in London and from the cold water supply of a further three practices. The prevalence of Legionella pneumophila antibodies was less than that seen in a comparable group of London blood donors.

\section{Conclusion}

The risk to dentists' health from potential exposure to legionellae in this cohort of dentists was very low and this was confirmed by the very low seroprevalence and antibody titres to legionella detected in the dentists.

\section{IN BRIEF}

- Compared with previously reported prevalence rates of legionella spp. in dental hospitals in Europe and North America the prevalence of legionellae in dental unit water samples taken from general dental practices in London and rural Northern Ireland was very low.

- A correspondingly low level of legionella antibody was detected in the recruited dentists, which were comparable to background levels in blood donors.

- It cannot be assumed that all GDP dental unit waterlines will be free of legionellae as environmental conditions may prevail in an individual surgery that promotes the growth of legionella.

- In order to provide safe DUWL water that complies with drinking water quality standard, dentists are advised to follow Health and Safety Commission and BDA infection control guidelines for water management.

\section{COMMENT}

This manuscript deals with the risk of dental personnel and patients to Legionnaires' disease associated with dental unit waterlines (DUWL). Legionellae are water borne microorganisms that have been linked to outbreaks of infection associated with cooling water systems, water fountains, spa pools and hot and cold water systems. Legionella pneumophila has been shown to persistently colonise hospital water supplies and cause recurrent clusters of nosocomial infection.

Typically legionellae enter the DUWL from mains drinking water and are able to proliferate in the favourable microhabitat provided by biofilms in DUWL plumbing systems. DUWL in dental hospitals have themselves been associated with higher recovery rates of legionellae. The authors in this paper studied the presence of legionellae in DUWL in general dental practices in London (166) and rural Northern Ireland (100). Legionella was not recovered in any of the surgeries in Northern Ireland and was only recovered in $1(0.37 \%)$ out of 166 surgeries in London indicating a very low prevalence. In this study $80 \%$ of the DUWL were directly fed by mains water and as such there was no requirement for large storage tanks, as found in institutional buildings, that may otherwise act as a reservoir for repeated seeding.

The manuscript also investigated the prevalence of $L$ pneumophila antibodies in blood samples from the dentists. Previous studies had demonstrated that $34 \%$ of the dentists sampled were seropositive for legionellae. In this study only one dentist was seropositive and the prevalence of L pneumophila antibodies in this study's population of dentists did not exceed the background level seen in a London blood donor control group.

This study demonstrates that dentists and patients in general dental practises in London and Northern Ireland would be at a minimal risk of serious respiratory illness due to legionella. However, dentists should act with due diligence in the implementation of a water management guidance regime as described by the Health and Safety Commission's Approved Code of Practice on the control of legionella in water systems.

J. Walker,

President of the International Biodet. and Biodegrad. Soc. CAMR, Porton Down, Salisbury doi:10.1038/sj.bdj.4810732 